\title{
Upregulation of Mir-34a in AGS Gastric Cancer Cells by a PLGA-PEG-PLGA Chrysin Nano Formulation
}

\author{
Farideh Mohammadian ${ }^{1,4}$, Alireza Abhari², Hassan Dariushnejad ${ }^{1,3}$, Faraz \\ Zarghami $^{2}$, Alireza Nikanfar ${ }^{3}$, Yones Pilehvar-Soltanahmadi ${ }^{1}$, Nosratollah \\ Zarghami $^{3 *}$
}

\begin{abstract}
Background: Nano-therapy has the potential to revolutionize cancer therapy. Chrysin, a natural flavonoid, was recently recognized as having important biological roles in chemical defenses and nitrogen fixation, with anti-inflammatory and anti-oxidant effects but the poor water solubility of flavonoids limitstheir bioavailability and biomedical applications. Objective: Chrysin loaded PLGA-PEG-PLGA was assessed for improvement of solubility, drug tolerance and adverse effects and accumulation in a gastric cancer cell line (AGS). Materials and Methods: Chrysin loaded PLGA-PEG copolymers were prepared using the double emulsion method (W/O/W). The morphology and size distributions of the prepared PLGA-PEG nanospheres were investigated by $1 \mathrm{H}$ NMR, FT-IR and SEM. The in vitro cytotoxicity of pure and nano-chrysin was tested by MTT assay and miR-34a was measured by real-time PCR. Results: 1H NMR, FT-IR and SEM confirmed the PLGA-PEG structure and chrysin loaded on nanoparticles. The MTT results for different concentrations of chrysin at different times for the treatment of AGS cell line showed IC50 values of 68.2, 56.2 and $42.3 \mu \mathrm{M}$ and 58.2, 44.2,36.8 $\mu \mathrm{M}$ after 24, 48, and 72 hours of treatment, respectively for chrysin itslef and chrysin-loaded nanoparticles. The results of real time PCR showed that expression of miR-34a was upregulated to a greater extent via nano chrysin rather than free chrysin. Conclusions: Our study demonstrates chrysin loaded PLGA-PEG promises a natural and efficient system for anticancer drug delivery to fight gastric cancer.
\end{abstract}

Keywords: Chrysin - PLGA-PEG - MTT assay - gastric cancer - miR-34a

Asian Pac J Cancer Prev, 16 (18), 8259-8263

\section{Introduction}

Gastric cancer is third most common cause of cancerrelated mortality in the world (Okuchukwu and Olayiwola, 2015). Despite of many advances for gastric cancer therapy, unfortunately prognosis for gastric cancer patients still remain unsatisfying. After surgery, chemotherapy play important role in the treatment of gastric cancer but chemotherapeutic drugs shows less validation and security. Therefore it is necessary to search and find new agents and delivery systems for providing new drugs that they posses more clinical benefits and better outcomes for gastric cancer therapy (Fock, 2014).

Recent studies have shown that natural agents have chemopreventive properties and therapeutic potential and the other hand they are relatively nontoxic (Basmadjian et al., 2014). One of such agent is chrysin. Chrysin (5,7-dihydroxy flavones) is a biologically active flavones extracted from honey, bee propolis and plants. It has been shown to have antioxidant, anti-inflammatory properties and anticancer effects by inhibiting cell proliferation and induction of cell death in variety of cancer cells (Kasala et al., 2015).

Micro RNAs (miRNAs) are endogenous, single strand and non-coding 20-22 nucleotides small RNAs that regulate gene expression via mRNA degradation or translational repression (Wang et al., 2015). Disregulation of genes, specifically proto-oncogenes and tumorsuppressor genes, results in abnormal function or expression of oncogenic and tumor suppressor proteins. Evidences indicate that microRNAs are involved in important biological processes related to proliferation, differentiation, apoptosis, angiogenesis, metastasis and immune response. Disregulation of these processes lead to cancer initiation, progression and treatment outcomes. It has been shown that expression of miRNAs to be related to gastric cancer as well as other cancers and play crucial roles in regulating cancer related genes (Samarghandian et al., 2011; Hur et al., 2013).

The poor water solubility of flavonoids especially chrysin is one of the obstacle that limit using this agent in biomedical applications (Kwon et al., 2010; Munin and

${ }^{1}$ Department of Medical Biotechnology, Faculty of Advance Medical Sciences, ${ }^{2}$ Department of Clinical Biochemistry, Faculty of Medicine, ${ }^{3}$ Hematology and Oncology Research Center, Tabriz University of Medical Sciences, ${ }^{4}$ International Aras Branch, University of Medical Sciences, Tabriz, Iran*For correspondence: zarghami@tbzmed.ac.ir 
Edwards-Lévy, 2011).

When drug loaded polymeric nanoparticles, they show better pharmacological structures for the accumulation in tumor tissue or cells, improved retention effect and permeability. These properties can improve drug tolerance and decrease adverse effects (Ghalhar et al., 2013; Zarouni et al., 2015). Also using of nanoparticles of biodegradable polymers have great potential to solve most of the major problems encountered in using flanovids in therapy (Nejati-Koshki et al., 2013).

PLGA (poly lactic-co-glycolid acid) is polymeric nanoparticle that possesses high biocompatibility and biodegradability. PLGA safe for human and its safety were approved by U.S Food and Drug Administration (FDA) (Alimohammadi and Joo, 2014). Modification surface of PLGA with PEG (polyethylene glycol) improve permeability and half-life of circulation (Dwivedi et al., 2014).

In this study we evaluate effect chrysin and encapsulation form of chrysin in PLGA-PEG-PLGA nanoparticle in gastric cancer cell line.

\section{Materials and Methods}

\section{Materials}

Chrysin, PEG ${ }_{(6000)}, 3$ (4,5-dimethylthiazol-2yl)2,5-diphenil-tetrazolium bromide (MTT), stannous octoate(Sn(Oct)2), dichloromethane(DCM), dimethyl sulphoxide(DMSO), polyvinyl alcohol(PVA) and D,LLactide were purchased from Sigma-Aldrich(USA). AGS gastric cancer cell line was obtained from Pasteur Institute of Iran. Trypsin-EDTA, fetal bovine serum (FBS), RPMI1640, penicillin G and streptomycin were from Gibco, Invitrogen(UK).

\section{Preparation of PLGA-PEG-PLGA triblock copolymer}

PLGA-PEG tri-block copolymer was synthesized via ring opening polymerization of DL-lactid and glycolide in presence of $\mathrm{PEG}_{6000}$. In presence of stannous octoate [SN (Oct)2] as catalyst, PLGA and PEG $_{6000}$ were polymerized. Melt polymerization procedure done under vacuum condition. PEG $_{6000} 1.54(45 \% \mathrm{~W} / \mathrm{W})$, DL-lactide(2.882gr) and glycolide $(0.570 \mathrm{gr})$ were melted in bottleneck flask under a nitrogen atmosphere and $140^{\circ} \mathrm{C}$ until melting was completed. Reaction mixture comprising a $3: 1$ proportion of DL-lactide to glycolide and $0.05 \%$ (W/W) $\mathrm{Sn}(\mathrm{Oct}) 2$ was prepared and heated to $180^{\circ} \mathrm{C}$ for next four hours.

Chrysin loading and Determination of entrapment efficiency (EE) and drug loading (DL)

$\mathrm{S} / \mathrm{O} / \mathrm{W}$ technique was used for loading chrysin in PLGA-PEG nanoparticls. Briefly, $20 \mathrm{mg}$ chrysin and 200 mg PLGA-PEG were added to solvent (dichloromethane). This solution sonicated for 1 minute to make the primary $\mathrm{S} / \mathrm{O}$ emulsion. Polyvinyl alcohol (PVA) $1 \%$ and dimethyl solphoxide (DMSO) $(1: 1)$ was added to $\mathrm{S} / \mathrm{O}$ emulsion then sonicated for another 1 minute to produce $\mathrm{S} / \mathrm{O} / \mathrm{W}$ emulsion. After this procedure, solvent were evaporated by rotary evaporator (Rotary Evaporator, Heidolph Instruments, Hei-VAP series). Subsequently this emulsion was centrifuged 30 minute at $10000 \mathrm{~g}$. Assessing the supernatant of centrifuged emulsion at $348 \mathrm{~nm}$ with spectrophotometer (Shimadzu) was used for measuring drug encapsulation efficiency. By the following formula, the percent of chrysin encapsulated on the nanoparticles (Equation 1) and drug loading (Equation 2) was measured:

\section{EE $=($ Weight of CHRYSIN in NP) / (Weight of the initial drug $) \times 100 \%$ (Equation 1) \\ $D L=($ Weight of CHRYSIN in NPS $) /($ Weight of NPS $) \times 100 \%$ (Equation 2)}

\section{HNMR spectrum of copolymer}

1HNMR spectrum has been used for structure analysis of copolymer. Spectra were recorded using Brucker $\mathrm{AM} 300.13 \mathrm{MHz}$ spectrometer (Germany) at $25^{\circ} \mathrm{C}$ and deuterated chloroform $(\mathrm{CDCl} 3)$ was used as solvent.

\section{FTIR analysis}

Functional group characterization was performed via FTIR analysis using Perklin Elmer Series (USA) FTIR spectroscopy. The FTIR spectrum was gained from sample spreading on the potassium bromide tablet.

\section{Scanning electron microscopy (SEM)}

Scanning electron microscopy (SEM) (KYKY model EM3200) was used for the surface morphology of the formulated nanoparticle. The lyophilized samples spread over conductive tape and fixed on metallic stud.

\section{Cell line and cell culture}

AGS (human gastric adenocarcinoma) was purchased cultured in RPMI-1640 medium supplemented with 10\% heat inactivated fetal bovine serum (FBS) with $2 \mathrm{mg} / \mathrm{ml}$ sodium bicarbonate, $0.05 \mathrm{mg} / \mathrm{ml}$ penicillin $\mathrm{G}$ and $100 \mu \mathrm{g} /$ $\mathrm{ml}$ streptomycin. The cell line was cultured in $25 \mathrm{~cm} 2$ flasks and maintained in a humidified incubator containing $5 \% \mathrm{CO}_{2}$ at $37^{\circ} \mathrm{C}$.

\section{In vitro cytotoxicity}

The cytotoxicity of treatments was determined by MTT assay. Just before treatments, AGS cell line was cultured in complete medium at a density of 7500 cells/ well in 96-well tissue culture plates (Nunc, Denmark) and incubated overnight at $37^{\circ} \mathrm{C}$. The next day, the culture medium was replaced with $200 \mu 1$ of fresh complete medium and then the cells were treated with different concentration of nano capsulated form of Chrysin. Treatments with RPMI without drug were also considered as a blank control. After $24 \mathrm{~h}$ of incubation, the culture medium was removed and the cells were incubated with an MTT solution $(0 / 2 \mathrm{mg} / \mathrm{ml}, 200 \mu \mathrm{l})$ for $4 \mathrm{~h}$ at $37^{\circ} \mathrm{C}$ in a humidified atmosphere. After an incubation period MTT solution was removed. Subsequently, $200 \mu 1$ of DMSO and $25 \mu 1$ of Sorenson's glycine buffer were added to dissolve the formazan crystals. Finally the absorbance was recorded in a microplate reader (Bio Tech Instruments, USA) at test wavelength of $570 \mathrm{~nm}$ with a reference wavelength of 650 $\mathrm{nm}$. The percentage of cell viability was determined using the following formula:

Cell viability $(\%)=$ Absorbance Test $/$ Absorbance Control $\times 100$ 
The IC50 value (concentration that induced 50\% cytotoxicity) was calculated using GraphPad Prism 6.01 software (GraphPad Software Inc, USA).

\section{RNA isolation}

Total RNA was isolated using Exiqon miRCURY RNA isolation kit (Exiqon, Denmark) according to the manufacturer's instructions. Quantity and quality of the isolated RNA were measured by Nanodrop 1000 (NanoDropND-1000spectrophotometer; Thermo Fisher Scientific, Waltham, MA). Total RNAs were reversed to cDNA using LNA universal RT miRNA PCR kit (Exiqon, Denmark). Briefly, $20 \mathrm{ng}$ of total RNA was reverse transcribed. cDNA synthesis was performed by thermal cycler (Eppendorf, Germany) with the following parameter values; $60 \mathrm{~min}$ at $42^{\circ} \mathrm{C}, 5 \mathrm{~min}$ at $95^{\circ} \mathrm{C}$ and immediately cooled to $4^{\circ} \mathrm{C}$ until use.

\section{Real-time PCR analysis}

Quantitative real-time reverse transcriptase-PCR was carried out using the Corbett Rotor-Gene 6000 Real-Time PCR system (Qiagen, Germany). MiRNAs quantification was performed using MiRCURY LNA Universal RT microRNA PCR system (Exiqon, Denmark). Mir-16 was used as the endogenous control miRNA.

\section{Statistical analysis}

Statistical analysis was performed using SPSS software (version 18). Comparison between data were performed by one-way ANOVA followed by Tukey,s HSD test. The results were expressed as mean \pm SEM. P-value $<0.05$ was considered as significant.

\section{Results}

\section{FTIR spectrum of PLGA-PEG-PLGA copolymer}

Successfully, PLGA-PEG-PLGA copolymer was synthesized by ring opening technique. As illustrated in the figure 1 the absorption peak at $3432.33 \mathrm{~cm}-1$ is assigned to terminal hydroxyl groups in the PLGA-PEG-PLGA. The band at $2915 \mathrm{~cm}-1$ attributed to the $\mathrm{C}-\mathrm{H}$ stretch. Bands at 1735 and $1625 \mathrm{~cm}-1$ due to $\mathrm{C}=\mathrm{O}$ stretches. A strong band at $1024 \mathrm{~cm}-1$ corresponds to the characteristic absorption of the $\mathrm{C}-\mathrm{C}, \mathrm{C}-\mathrm{O}$ and $\mathrm{C}-\mathrm{O}-\mathrm{C}$ stretches.

\section{HNMR spectrum of synthesized copolymer}

1HNMR spectrum was recorded to confirm the structure of the triblock copolymer. Spectrum of the PLGA-PEG-PLGA that show in figure 2. Is very similar to the previously reported spectrum (Chen et al., 2005). Tetramethylsaline (TMS) used as internal refrance and chemical shift $(\delta)$ was measured in ppm. Large peak at $3.65 \mathrm{ppm}$, attributed to the methylene group of the PEG. Overlapping doublets at $1.55 \mathrm{ppm}$ were corresponded to the methyl group of the D-lactic acid and L-lactic acid repeat units. Bond at $5.2 \mathrm{ppm}$ show the lactic acid $\mathrm{CH}$ and bond at $4.8 \mathrm{ppm}$ due to the glycolic acid $\mathrm{CH}$. High complexity of the 2 peaks resulting from different L-lactic, D-lactic and glycolic acid sequence in polymer structure.
Size and size distribution of nanoparticles

The surface morphology of the nanospheres was observed by SEM. The nanographs of PLGA-PEG-PLGA and chrysin loaded nanoparticles are shown in Fig3a and Fig3b respectively. As observed in the photograph, it can be seen that the nanoparticles were well aggregated due to nanosize of the PLGA-PEG-PLGA about 50 $\mathrm{nm}$. After encapsulation of chrysin, PLGA-PEG-PLGA nanoparticles, the size of particles alter to 70-300 nm. These SEM nanographs showed PLGA-PEG-PLGA nanoparticles were spherical in shape, uniform and had standard size.

Entrapment efficiency (EE) and drug loading (DL)

Based on equation 1 and 2, encapsulation efficiency

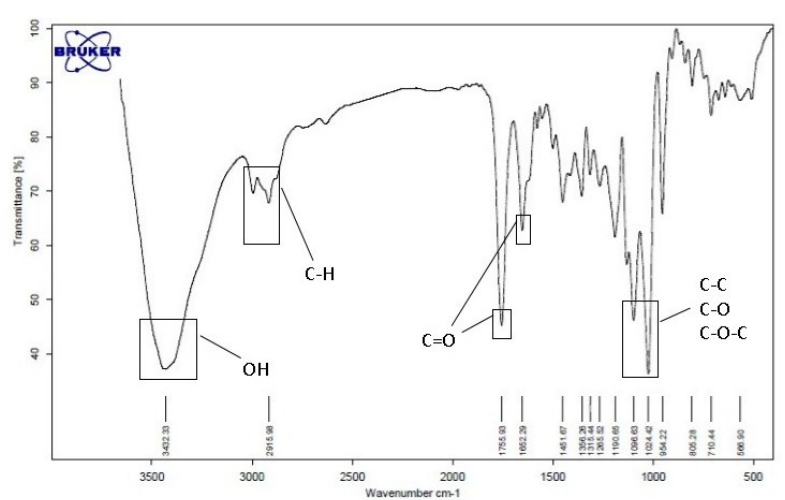

Figure 1. IR spectrum of chrysin loaded in PLGAPEG-PLGA

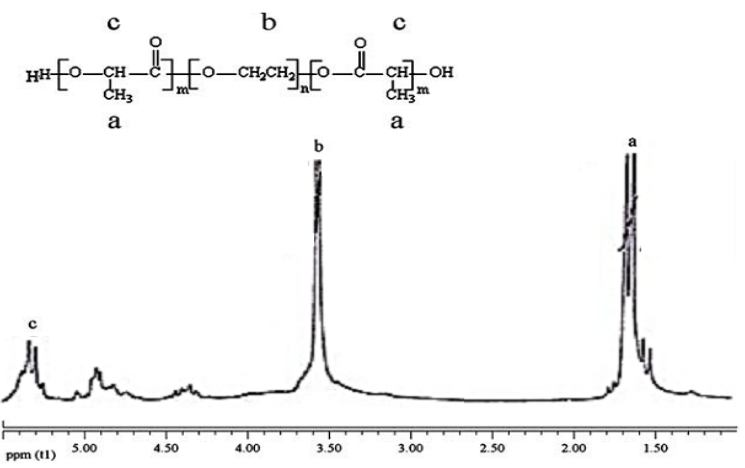

Figure 2. 1H NMR Spectrum of PLGA-PEG-PLGA Co-polymer
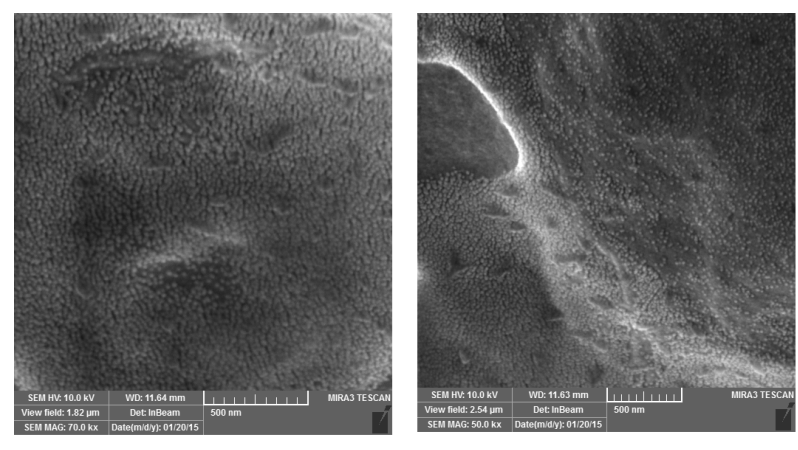

a

Figure 3. SEM Picture Show (a) the size PLGA-PEGPLGA and (b) Chrysin Loaded in PLGA-PEG-PLGA Nanoparticle 
(EE) and drug loading (DL) was $98.74 \%$ and $16.13 \%$ respectively. Existence hydrophobic groups (such as GA and D,L-LA) in the kernel of PLGA-PEG-PLGA micelles made chrysin easily load in nanoparticle by interaction between chrysin and hydrophobic groups.

\section{Cell cytotoxicity effect}

MTT assay performed to analyze cytotoxicity effect of pure chrysin and chrysin loaded PLGA-PEG-PLGA $(0-160 \mu \mathrm{M})$ for 24,48 and $72 \mathrm{~h}$ on AGS gastric cancer cell line .results suggested that chrysin and chrysin loaded in nanoparticle inhibit cell growth in dose-dependent manner. The half maximal inhibitory concentration (IC50) of nano chrysin was 58.24 after $24 \mathrm{~h}$ (fig $4 \mathrm{a}$ ), 44.21 at $48 \mathrm{~h}$ (fig4b) and decline to 36.8 after $72 \mathrm{~h}$ (fig4c). However, free chrysin show 68.24 after $24 \mathrm{~h}, 56.16$ after $48 \mathrm{~h}$ and 42.32 after $72 \mathrm{~h}$. In the other hand no inhibitory effect was observed for free PLGA-PEG-PLGA (data not shown). In total, these findings demonstrate that PLGA-PEG-PLGA
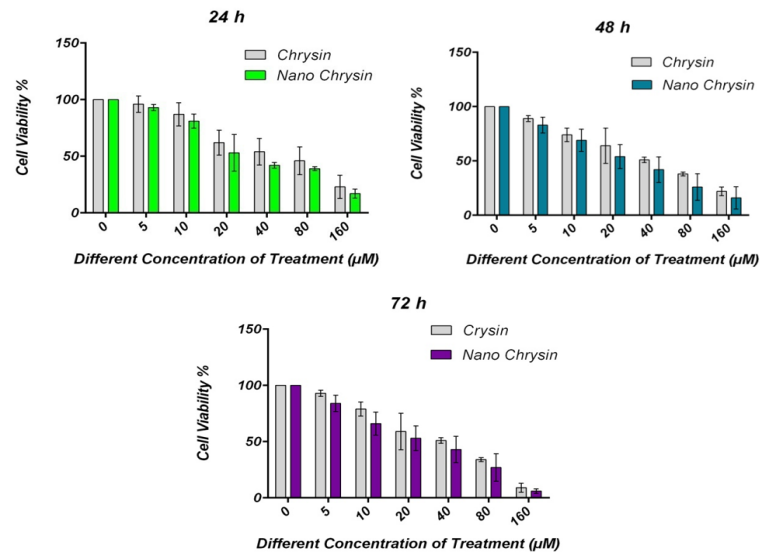

Figure 4. Cytotoxicity Effect of Pure Chrysin and Chrysin loaded in PLGA-PEG-PLGA on AGS cell for 24h; 48h and 72h Exposure
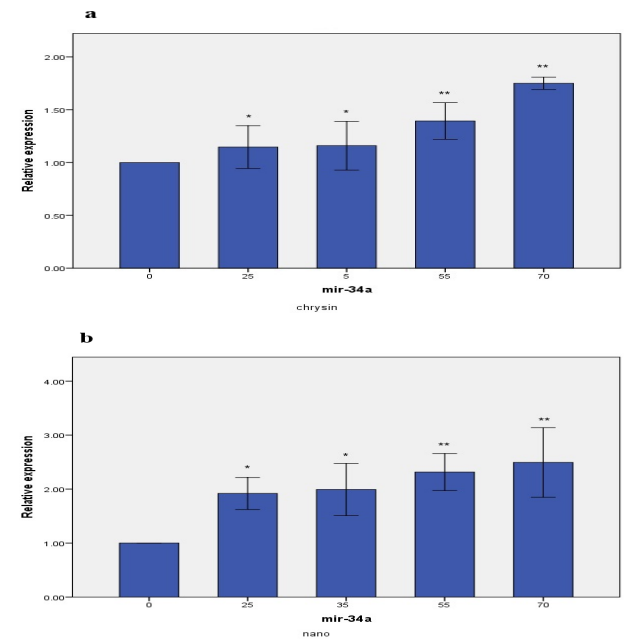

Figure 5. Effects of Pure Chrysin (a) and Chrysin Loaded in PLGA-PEG-PLGA (b) on the Up-regulation of miR-34a after 24-hour Treatment with 0, 25, 35, 55 and $70 \mu \mathrm{M}$ free agent and Chrysin Loaded in PLGAPEG-PLGA. Expression of miR-34a Significantly Increased at 55 and $70 \mu \mathrm{M}$ Concentrations. Data expressed as mean \pm SEM; $* \mathrm{P}<0.05$ and $* * \mathrm{P}<0.01$ increased the water solubility and entry of chrysin to cells without any toxic effects regarding PLGA-PEG-PLGA as a carrier (table 1).

\section{Quantitative Real-Time PCR}

The effect of pure chrysin and chrysin loaded in PLGA-PEG-PLGA on the expression of miR-34a was investigated by Quantitative Real-Time PCR. miR-34a mRNA levels were normalized by miR-16. AGS cells exposed to different concentration of free agent and chrysin loaded nanoparticle in equal concentration $(0$, $25,35,55$ and $70 \mu \mathrm{M})$ for $24 \mathrm{~h}$. Data analysis showed that treatment with chrysin increase miR-34a expression. On the other hand nano capsulation form of chrysin shows more ability to restore mir-34a expression. As it was shown in figure 5 chrysin upregulate 2 fold and nano chrysin increased 3 fold of mir-34a expression at $70 \mu \mathrm{M}$ concentration in comparison to untreated control cell. Figure 5 show that expression of miR-34a significantly increased at 55 and $70 \mu \mathrm{M}$ concentrations (p value less than 0.001).

\section{Discussion}

Spite of advances in cancer therapy, gastric cancer still health problem in the world and treatment of this malignancy is dependent mainly on conventional cytotoxic chemotherapy (Lee et al., 2013). This disease requirement for new therapies that is able to treat this malignancy. Natural agents and medical plants have been demonstrated to be a source of effective anticancer agents (Basmadjian et al., 2014). Chrysin is phenolic compound and can be finding in honey. This agent show anticancer effects and induction of apoptosis in some cancer cells (Khoo et al., 2010). But molecular mechanism of chrysin not clearly understood and need more research.

MiR-34a belongs to the miR-34 family and involved in the P53 tumor suppressor network. Recently it was reported that miR-34a was down regulated in gastric cancer (Hu et al., 2014). it seems that restoration of miR$34 \mathrm{a}$ is able to reconstruct tumor suppressing signaling pathway in this cancer ( Ji et al., 2008). Our data suggest the chrysin can restore miR-34a expression and may hold significant promise as a novel molecular therapy for human gastric cancer.

Now a day, application of nanothecnology in drug delivery is one of the most promising tools to treat cancer. The extraordinary properties of PLGA-PEG-PLGA make this nanoparticle favorable for drug delivery and safety of this nananoparticle approved by FDA.( refrance bala zadi). In this study, PLGA-PEG-PLGA nanoparticle was used to increase crysin solubility. FTIR, 1HNMR and SEM analysis confirmed chrysin loaded in PLGA-PEGPLGA properly. MTT assay showed that chrysin loaded in PLGA-PEG-PLGA had more cytotoxicity effect rather than pure form.

Boon Yin Khoo et al. for developing the effectiveness of chrysin used phosphorylated form this agent (Zhang et al., 2004). While we used chrysin loaded in PLGAPEG-PLGA nanoparticle to improve the solubility and anticancer effect of this flavonid. 
In conclusion, chrisin encapsulated with PLGA-PEGPLGA show significant inhibitory effect in cell growth in comparison with pure chrysin and this agent can restore miR-34a expression. Our study show, chrysin loaded in PLGA-PEG-PLGA is promising approach for gastric cancer therapy.

\section{Acknowledgements}

Authors would like to thank Hematology and Oncology Research Center, Tabriz University of Medical Sciences, Tabriz, Iran for supporting this project (grant No 92/34), which was a part of thesis No 92/1-1/1.

\section{References}

Alimohammadi YH, Joo SW (2014). PLGA-based nanoparticles as cancer drug delivery systems. Asian Pac J Cancer Prev, 15, 517-35.

Basmadjian C, Zhao Q, Bentouhami E, et al (2014). Cancer wars: natural products strike back. Frontiers in chemistry, 2 .

Chen S, Pieper R, Webster DC, et al (2005). Triblock copolymers: synthesis, characterization, and delivery of a model protein. International journal of pharmaceutics, 288, 207-18.

Dwivedi M, Sharma S, Shukla P, et al (2014). Development and Evaluation of Anticancer Polymeric Nano-Formulations Containing Curcumin and Natural Bioenhancers. Journal of Biomaterials and Tissue Engineering, 4, 198-202.

Fock K (2014). Review article: the epidemiology and prevention of gastric cancer. Alimentary pharmacology \& therapeutics, 40, 250-60.

Ghalhar MG, Akbarzadeh A, Rahmati M, et al (2013). Comparison of Inhibitory Effects of 17-AAG Nanoparticles and Free 17-AAG on HSP90 Gene Expression in Breast Cancer. Asian Pacific journal of cancer prevention: APJCP, 15, 7113-8.

Hu C-E, Liu Y-C, Zhang H-D, et al (2014). The RNA-binding protein PCBP2 facilitates gastric carcinoma growth by targeting miR-34a. Biochemical and biophysical research communications, 448, 437-42.

Hur K, Toiyama Y, Takahashi M, et al (2013). MicroRNA-200c modulates epithelial-to-mesenchymal transition (EMT) in human colorectal cancer metastasis. Gut, 62, 1315-26.

Ji Q, Hao X, Meng Y, et al (2008). Restoration of tumor suppressor miR-34 inhibits human p53-mutant gastric cancer tumorspheres. BMC cancer, 8, 266.

Kasala ER, Bodduluru LN, Madana RM, et al (2015). Chemopreventive and therapeutic potential of chrysin in cancer: mechanistic perspectives. Toxicology Letters, 233, 214-25.

Khoo BY, Chua SL, Balaram P (2010). Apoptotic effects of chrysin in human cancer cell lines. International J Molecular Sci, 11, 2188-99.

Kwon Y, Kim H, Park S, et al (2010). Enhancement of solubility and antioxidant activity of some flavonoids based on the inclusion complexation with sulfobutylether $\beta$-cyclodextrin. Bull Korean Chem Soc, 31, 3035-7.

Lee HJ, Song I-C, Yun H-J, et al (2013). CXC chemokines and chemokine receptors in gastric cancer: From basic findings towards therapeutic.

Munin A, Edwards-Lévy F (2011). Encapsulation of natural polyphenolic compounds; a review. Pharmaceutics, $\mathbf{3}$, 793-829.

Nejati-Koshki K, Akbarzadeh A, Pourhasan-Moghadam M, et al (2013). Inhibition of leptin and leptin receptor gene expression by silibinin-curcumin combination. Asian Pac
$J$ Cancer Prev, 14, 6595-9.

Okuchukwu EH, Olayiwola OA (2015). Epidemiology and clinicopathological characteristics of gastric cancer--the Nigerian setting in view. Niger J Med, 24, 71-80.

Samarghandian S, Afshari JT, Davoodi S (2011). Chrysin reduces proliferation and induces apoptosis in the human prostate cancer cell line pc-3. Clinics, 66, 1073-9.

Wang Q-X, Zhu Y-Q, Zhang H, et al (2015). Altered MiRNA Expression in Gastric Cancer: a Systematic Review and Meta-Analysis. Cellular Physiology and Biochemistry, 35, 933-44.

Zarouni M, Salehi R,Akbarzadeh A, et al (2015). Biocompatible Polymer Coated Paramagnetic Nanoparticles for Doxorubicin Delivery: Synthesis and Anticancer Effects Against Human Breast Cancer Cells. International Journal of Polymeric Materials and Polymeric Biomaterials, 64, 718-26.

Zhang T, Chen X, Qu L, et al (2004). Chrysin and its phosphate ester inhibit cell proliferation and induce apoptosis in Hela cells. Bioorganic \& medicinal chemistry, 12, 6097-105. 